Quantitative other

\section{A local public campaign reduces outpatient antibiotic prescribing in Italy}

$10.1136 /$ eb-2013-101628

\section{Nick Francis}

Institute of Primary Care and Public Health, School of Medicine, Cardiff University, Cardiff, UK

Correspondence to Nick Francis, Institute of Primary Care and Public Health, School of Medicine, Cardiff University, 5th Floor, Neuadd Meirionnydd, Heath Park, Cardiff, CF14 4YS, UK; francisna@cf.ac.uk

Commentary on: Formoso G, Paltrinieri B, Marata AM, et al. Feasibility and effectiveness of a low cost campaign on antibiotic prescribing in Italy: community level, controlled, nonrandomised trial. BMJ 2013;347:f5391.

\section{Implications for practice and research}

- Public health campaigns can reduce antibiotic prescribing, although the effect is likely to be mediated primarily through changes in clinician behaviour rather than patient-consulting behaviour or expectations for antibiotics.

- More research is needed on the key components of antimicrobial stewardship activities: the effects on antimicrobial resistance, the costeffectiveness and the sustainability of effect.

\section{Context}

Antimicrobial resistance (AMR) is an important and growing international health threat. ${ }^{1}$ Exposure to antibiotics is the primary driver of AMR; therefore, efforts to tackle this problem commonly focus on reducing unnecessary prescribing. Most antibiotic prescribing occurs in primary care, and the majority of prescribing is for respiratory tract infections and other 'minor' infections where antibiotics confer little, if any, benefit. ${ }^{2}$ Because patient expectations for antibiotic treatment are often cited as a reason for prescribing in primary care, public health information campaigns have been used to try and modify public perceptions about the need for antibiotics. This study aimed to evaluate a local public information campaign in the north of Italy.

\section{Methods}

A public health campaign, focusing mainly on the use of antibiotics for upper respiratory tract infections, was run over a 4-month period in two provinces in northern Italy. Other provinces within the same region were used as a control group. Randomisation was not used to select provinces. The campaign's key messages were developed by a group of local doctors and the campaign involved posters, brochures, mass media and a local newsletter. Public knowledge and attitudes relating to the campaign's messages were assessed via telephone and internet surveys conducted by a polling agency in the intervention and control provinces. The average antibiotic prescribing rate for the 4-month intervention period and the following 1-month in intervention and control areas was calculated using data from regional prescribing databases. Generalised linear mixed models were used to compare health district level prescribing in intervention and control areas (11 in the intervention area and 31 in the rest of the region), taking into account baseline prescribing.

\section{Findings}

There was a reduction in antibiotic prescribing during the evaluation period compared with the same period the year before in intervention (11.9\% reduction; from 22.7 to 20 defined daily doses/1000 inhabitants per day) and control (7.4\% reduction; from 22.7 to 21 ) areas. Using a linear regression statistical model of health district-level data, they found a $4.3 \%(95 \% \mathrm{CI}-7.5 \%$ to $-1.5 \%)$ reduction in prescribing in the intervention area compared with the control area. Recall of campaign slogans and graphics was similar in both areas. Knowledge and attitudes consistent with campaign messages worsened in both areas.

\section{Commentary}

This study had some methodological weaknesses, but the results suggest a small reduction in antibiotic prescribing during the 5-month evaluation period as a result of the intervention. This is consistent with previous evaluations of public campaigns on appropriate use of antibiotics, which have had mixed results but mostly demonstrate small reductions in prescribing. ${ }^{3}$ Public campaign interventions are complex interventions with many components, many possible mechanisms of action and many possible mediating factors, which make it difficult to determine the 'active ingredients' and how different elements interact or might work differently in different settings.

Interestingly, the lack of public awareness of the campaign materials and reduction in knowledge and attitudes seen in this study (consistent with some previous campaigns) suggests that the effect is likely to be mediated though changes in the behaviour of clinicians rather than the public. This makes some sense, as ultimately it is clinicians who prescribe antibiotics, and although patient pressure can influence prescribing, more often it is clinicians' incorrect assumptions about patient expectations that influence prescribing. ${ }^{4}$

Important questions remain about the sustainability of the effect and the cost-effectiveness of interventions such as this. The true costs of AMR are difficult to calculate but are likely to be very large indeed ${ }^{5}$; therefore, even small reductions in prescribing may be cost-effective. However, interventions to reduce unnecessary prescribing almost certainly need to be multifaceted and sustained over longer periods of time to have a lasting effect, and impact on resistance is not yet clear.

Competing interests None.
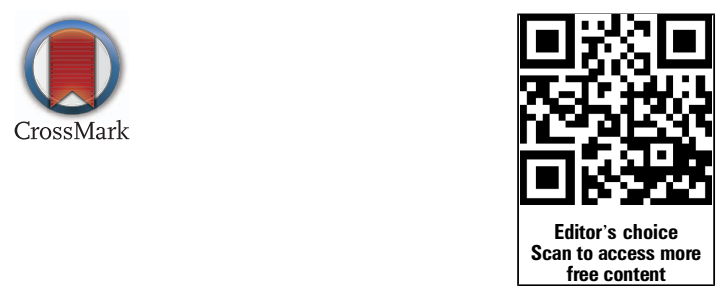

\section{References}

1. Davies SC. Annual report of the Chief Medical Officer, volume two, 2011, infections and the rise of antibiotic resistance. London: Department of Health, 2013.

2. National Institute of Health and Care Excellence. Respiratory tract infectionsantibiotic prescribing: prescribing of antibiotics for self-limiting respiratory tract infections in adults and children in primary care. London, UK: National Institute for Health and Care Excellence, 2009:1-121.

3. Huttner B, Goossens H, Verheij TJM, et al. Characteristics and outcomes of public campaigns aimed at improving the use of antibiotics in outpatients in high-income countries. Lancet Infect Dis 2010;10:17-31.

4. Coenen S, Francis NA, Kelly M, et al. Are patient views about antibiotics related to clinician perceptions, management and outcome? A multi-country study in outpatients with acute cough. PLOS ONE 2013;8:e76691.

5. Smith R, Coast J. The true cost of antimicrobial resistance. BMJ 2013;346:f1493. 